\title{
X-ray Emission from Massive Stars at the Core of Very Young Clusters
}

\author{
Norbert S. Schulz \\ Kavli Institute of Astrophysics and Space Research, Massachusetts Institute of Technology, \\ NE83-565, One Kendall Square, Bldg. 300, Cambridge 02139, USA \\ email: nss@space.mit.edu
}

\begin{abstract}
Most cores of very young stellar clusters contain one or more massive stars at various evolutionary stages. Observations of the Orion Nebula Cluster, Trumpler 37, NGC 2362, RCW38, NGC 3603 and many others provide the most comprehensive database to study stellar wind properties of these massive cluster stars in X-rays. In this presentation we review some of these observations and results and discuss them in the context of stellar winds and possible evolutionary implications. We argue that in very young clusters such as RCW38 and M17, shock heated remnants of a natal shell could serve as an alternate explanation to the colliding wind paradigm for the hot plasma components in the X-ray spectra.
\end{abstract}

Keywords. techniques: spectroscopic, stars: early-type, stars: winds, outflows, stars: formation

\section{Introduction}

The advent of Chandra and XMM-Newton allowed detailed X-ray diagnostics of X-ray stellar wind properties (Schulz et al. (2000), Kahn et al. (2001), Waldron \& Cassinelli (2001)). A decade of Chandra observations of high resolution X-ray spectra produced a convincing correlation between X-ray line ionizations and optical spectral types of OB stars (Walborn, Nichols \& Waldron (2009)) asserting that X-rays in stellar winds are indeed connected to fundamental stellar wind parameters. However these observations also produced a zoo of other X-ray production mechanisms ranging from magnetically confined winds, colliding wind activites, as well as massive dense winds as was most recently reported on (Schulz et al. (2003), Gagne et al. (2005), Pollock \& Corcoran (2006), Huenemoerder et al. (2015)).

\section{Young Massive Cluster Stars}

Observations of clusters like the Orion Nebula Cluster (Schulz et al. (2001), Feigelson et al. (2005)), M17 (Townsley et al. (2003)) or the Carina region (Townsley et al. (2011)) have produced plenty of evidence for either magnetically confined winds in Orion, colliding winds systems in M17 and Carina. Observing X-rays from wind phenomena in very young dense clusters require high spatial resolution and effective areas leaving only a very few dozen clusters within the reach of Chandra.

Observations. In order to diagnose X-ray wind phenomena we need high spectral resolution to resolve X-ray line emissions. This reduces available clusters with reasonable Chandra exposures to less than a dozen objects. Table 1 shows about half a dozen of massive stars we have observed with sufficient exposures with the high energy transmission gratings (HETG) onboard Chandra. Key here is the fact that we associate the age of the massive star with the age of the cluster. There are three groups to consider. Very young massive stars with ages less the $1 \mathrm{Myr}$ include the Orion Trapezium (not shown), 


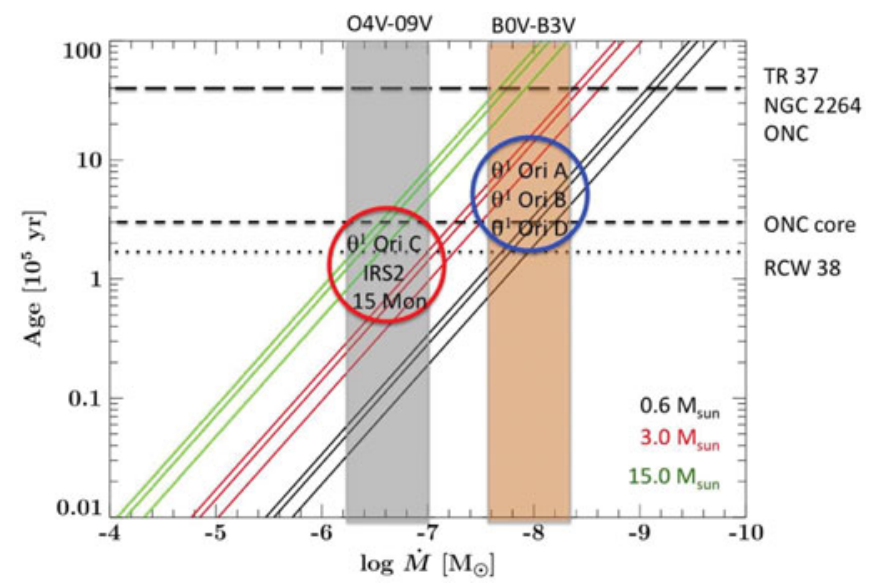

Figure 1. Photoevaporation rates of ultracompact H II envelopes in very young massive stellar winds. The plot shows rate curves for several natal cloud masses, the curves show the range of rate for various stellar mass loss rates, final wind velocities, and UV radiation fields. The black curves refer to $0.6 \mathrm{M}_{\odot}$ envelope mass, the red curves to $3 \mathrm{M}_{\odot}$ envelope mass, the green curves to $15 \mathrm{M}_{\odot}$ envelope mass.

Table 1. Properties of massive stars in very young clusters, young clusters, as well as evolved field stars

\begin{tabular}{|c|c|c|c|c|c|c|c|c|c|c|c|}
\hline Cluster & $\begin{array}{l}\text { Age } \\
{[\mathrm{Myr}]}\end{array}$ & Star & Туре & $\begin{array}{l}\mathrm{N}_{H} \\
{[(1)]}\end{array}$ & $\mathrm{A}_{V}$ & $\mid \begin{array}{c}\mathbf{L}_{x} \\
{\left[\operatorname{erg~s}^{-1}\right]}\end{array}$ & $\mathrm{L}_{x} / \mathrm{L}_{b o l}$ & $\begin{array}{l}\mathrm{kT}_{1} \\
{[\mathrm{MK}]}\end{array}$ & $\begin{array}{l}\mathrm{kT}_{2} \\
{[\mathrm{MK}]}\end{array}$ & $\begin{array}{l}\mathrm{EM}_{1} \\
{[(2)]}\end{array}$ & $\begin{array}{l}\mathrm{EM}_{2} \\
{[(2)]}\end{array}$ \\
\hline $\begin{array}{l}\text { Tr37 } \\
\text { NGC } 2362 \\
\text { NGC } 2264 \\
\text { RCW } 38 \\
\text { M } 17\end{array}$ & $\begin{array}{c}4 \\
4-5 \\
<2 \\
<0.2 \\
<1\end{array}$ & $\begin{array}{l}\text { HD 206067 } \\
\tau \text { CMA } \\
15 \text { Mon } \\
\text { IRS2 } \\
\text { KL Main } \\
\text { KL Comp }\end{array}$ & $\begin{array}{c}\mathrm{O} 6.5 \mathrm{~V}+09.5 \mathrm{~V} \\
\mathrm{O} 9 \mathrm{III} \\
\mathrm{O} 7 \mathrm{~V}+\mathrm{B} 2 \mathrm{~V} \\
2 \mathrm{O} 4-5.5 \mathrm{~V} \\
\mathrm{O} 4 \mathrm{~V} \\
\mathrm{O} 4 \mathrm{~V}\end{array}$ & $\begin{array}{c}4.9 \\
2.1 \\
1.6 \\
22.3 \\
10,5 \\
12.9\end{array}$ & \begin{tabular}{|c|}
1.56 \\
0.31 \\
0.22 \\
15 \\
13.2 \\
10.2
\end{tabular} & $\begin{array}{l}2.7 \times 10^{33} \\
2.9 \times 10^{33} \\
2.0 \times 10^{32} \\
3.7 \times 10^{33} \\
1.8 \times 10^{33} \\
1.2 \times 10^{33}\end{array}$ & $\begin{array}{l}-5.8 \\
-7.0 \\
-6.6 \\
-5.5 \\
-6.0 \\
-6.2\end{array}$ & $\begin{array}{l}1.6 \\
2.2 \\
1.9 \\
2.1 \\
9.0 \\
8.8\end{array}$ & $\begin{array}{c}7.0 \\
9.1 \\
7.4 \\
15.5 \\
87.5 \\
40.8\end{array}$ & $\begin{array}{c}21.6 \\
7.8 \\
3.3 \\
0.7 \\
4.4 \\
5.2\end{array}$ & $\begin{array}{c}0.7 \\
0.6 \\
0.3 \\
10.4 \\
12.2 \\
4.0\end{array}$ \\
\hline & $>5$ & $\zeta \mathrm{Pup}$ & $\mathrm{O} 4.5 \mathrm{I}$ & 5.3 & 0.19 & $1.0 \times 10^{33}$ & -6.6 & 3.7 & 8.0 & 3.9 & 0.4 \\
\hline NGC 3603 & $\begin{array}{l}<2.5 \\
>5\end{array}$ & $\begin{array}{l}\text { HD97950C } \\
\text { WR6 }\end{array}$ & $\begin{array}{l}\text { WN6h } \\
\text { WN4 }\end{array}$ & $\begin{array}{c}10.0 \\
2.1\end{array}$ & & $\mid \begin{array}{l}5.6 \times 10^{33} \\
7.9 \times 10^{32}\end{array}$ & -6.3 & $\begin{array}{l}5.5 \\
8.0\end{array}$ & $\begin{array}{l}48.3 \\
50.1\end{array}$ & $\begin{array}{c}12.4 \\
24\end{array}$ & $\begin{array}{c}13.6 \\
11\end{array}$ \\
\hline
\end{tabular}

Notes: (1) $10^{21} ;(2) 10^{55} \mathrm{~cm}^{-3}$

IRS2 in RCW38, and the two Kleinmann stars in M17. Young stars with ages of up to about 5 Myr include HD 206267 in Trumpler 37, $\tau$ CMa in NGC 2362, and 15 Mon in NGC 2264. Finally there are evolved field stars arguably with ages above $5 \mathrm{Myr}$ such as $\zeta$ Pup and WR6. Except for HD 97950C in the starburst cluster NGC 3603 these are stars where we have high signal to noise HETG spectra available.

Photoevaporation rates. Very young massive cluster stars at these young ages, even though they have already been on the main sequence for quite some time, still have residual circumstellar mass stemming from the star formation process in some form. Massive stars entering the main sequence are still embedded in their ultra-compact H II clouds (Wood \& Churchwell (1989)). Depending on the type of the star and its wind properties in terms of mass loss rates and terminal wind velocity it takes between $10^{5} \mathrm{yr}$ up to over $10^{6} \mathrm{yr}$ to full ridden the system of the H II cloud material. And even though star is optically visible, residual cloud mass can provide various levels of absorption in X-rays. Hollenbach, Johnstone \& Shu (1993) descibe the photoevaporation process in some detail and for massive winds project: 

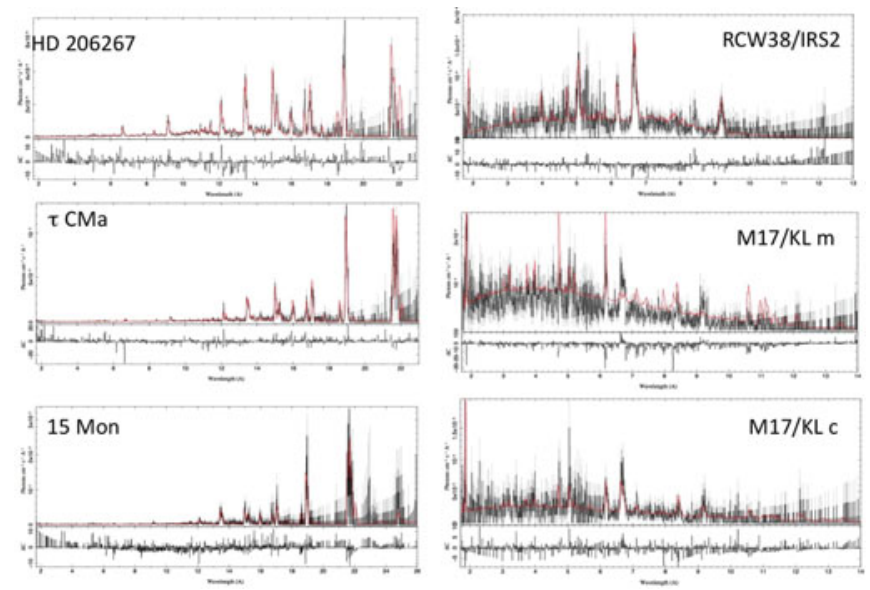

Figure 2. X-ray spectra of very young cluster stars (right panels) versus older cluster stars (left panels). The very young cluster stars are very hard and hot, the older ones conform with the standard wind paradigm independent of binary nature.

$$
\dot{M}_{p h} \simeq 5 \times 10^{-6} \frac{\dot{M}_{w}}{\left[10^{-7} M_{\odot}\right]} \frac{v_{w}}{\left[1000 \mathrm{~km} \mathrm{~s}^{-1}\right]}\left(\frac{\mathcal{N}_{U V}}{\left[10^{49} \mathrm{~s}^{-1}\right]}\right)^{-1 / 2} M_{\odot} \mathrm{yr}^{-1},
$$

where $\dot{M}_{p h}$ is the photoevaporaten rate, $\dot{M}_{w}$ the mass loss rate if the stellar wind, $v_{w}$ the wind velocity, and $\mathcal{N}_{\mathcal{U V}}$ the radiation field of the star. In Fig. 1 we plot the resulting photoevporation time versus the stellar massloss rate of the wind for various wind velocities and radiation fields for $\mathrm{H}$ II clouds of various masses.

Predictions for young clusters. Marked in Fig. 1 are mass loss ranges for various $\mathrm{O}$ and B-star regimes as well as ages for the clusters in Tab. 1. Massive stars in the red circle indicate cases for very young O-stars. While the cases for $15 \mathrm{Mon}, \tau \mathrm{CMa}$, and HD 206267 with a cluster age of 2-4 Myr is quite clear, i.e. all residual material has long been removed, the cases of $\theta^{2}$ Ori A, and IRS2, and the Kleinmann stars are ambiguous, i.e. residual material may still be in effect to some extent. In contrast, for the later type stars in the Orion Trapezium (see blue circle) it may be the case that these have not yet fully broken free and may not yet be visible at all in X-rays. Schulz et al. (2001) and Schulz et al. (2003) report that X-rays from these stars are unusually faint and suggest that their spectra resemble more coronal emissions from unseen low-mass companions. More careful extinction studies are necessary to make such an argument as the high $\mathrm{A}_{V} \mathrm{~s}$ we observe in RCW38 and M17 appear not so obvious in the Orion Trapezium stars.

\section{On the Origins of Hot X-ray Plasma}

One of the surprises in early Chandra observations of massive stars was the fact that some X-ray spectra show very hot (>20 MK) plasma components, some do not. One peculiar case is $\theta^{1}$ Ori $\mathrm{C}$ (Schulz et al. (2000)) in which a sizeable magnetic field compresses the wind into a dense circumstellar disk producing temperatures in excess of 50 MK (Gagne et al. (2005), Schulz et al. (2003)). However, claims for magnetically confined winds overall have so far not been substantiated in clusters other than Orion.

Colliding wind emission is another option since many of these cluster stars are multiple systems. This may also true for the two components of Kleinmann's star in M17, which in Tab. 1 are each listed as single O4V. Traditional colliding wind sources such as the 
$\eta$ Carina system (Corcoran et al. (2001)) or W140 (Pollock et al. (2005)) do show such hot plasma components but attached are also higher luminsities, a pattern of variability and specific X-ray line properties. Fig. 2 shows the HETG spectra of the young cluster stars from Tab. 1. The two temperature collisional plasma fit (red line in Fig. 2) to the spectra finds hot plasma components in the very young clusters stars, but not in the older cluster stars. The sample is certainly not statistically significant, but it is quite peculiar that binarity is a factor in both, very young and older cluster stars, but we do not detect hot plasmas in the latter. We also do not see excessive X-ray luminosities in these sources and within the data available no significant variability. The spectra also indicate very broad and skewed line widths of up to $3000 \mathrm{~km} \mathrm{~s}^{-1}$. Though all this does not rule out colliding wind activity in these systems, it raises the question whether the hot component in young stars might be of a different origin.

As we have shown above, because of the youth of these systems we have to anticipate the existence of remnants of natal shell material from the star formation process. Here we may invoke the wind model proposed by Owocki, Castor \& Rybicki (1988) which features reverse shocks that decelerate material as it rams into dense clumps. Located close to the terminal velocity of the wind, a shocked H II plasma at wind velocities of $2000 \mathrm{~km}$ $\mathrm{s}^{-1}$ would produce between 40 and $70 \mathrm{MK}$ depending on the degree of ionization of the shocked material. Emission measures as well as column densities can produce consistent plasma volumes and densities but require a high degree of clumpyness, i.e. a low fraction of sufficiently dense clumps are suspended in the shell volume. Residual shell material from the natal cloud can contribute to the hot plasma in very young massive stars but there are many details that need to be worked out to make a valid case.

\section{References}

Corcoran, M. F., Swank, J. H., Petre, R., Ishibashi, K., Davidson, K., Townsley, L., Smith, R., White, S., Viotti, R., \& Damelli, A. 2001, ApJ, 562, 1031

Feigelson, E. D., Getman, K., Townsley, L., Garmire, G., Preibisch, T., Grosso, N., Montmerle, T., Muench, A., \& McCaughrean, M. 2005, ApJS, 160, 379

Gagne, M., Oksala, M. E., Cohen, D. H., Tonnesen, S. K., ud-Doula, A., Owocki, S. P., Townsend, R. H. D., \& MacFarlane, J. J. 2005, ApJ, 628, 986

Hollenbach, D., Johnstone, D., \& Shu, F. 1993, in: J.P. Cassinelli \& E. Churchwell (eds.), Astronomy of the Pacific Conference Series, 35, 26

Huenemoerder, D. P., Gayley, K. G., Hamann, W.-R., Ignace, R., Nichols, J. S., Oskinova, L. Pollock, A. M. T., Schulz, N. S., \& Shenar, T. 2015, ApJ, 815, 29

Kahn, S. M., Leutenegger, M., Cottam, J. Rauw, G., Vreux, J. M., den Boggende, T., Mewe, R \& Guedel, M. 2001, A\&A, 365, L312

Owocki, S. P., Castor, J. L., \& Rybicki, G. B. 1988, ApJ, 335, 914

Pollock, A.M.T., Corcoran, M.F., Stevens, I.R. \& Williams, P.M. 2005 ApJ, 629, 482

Pollock, A. M. T. \& Corcoran, M. F. 2006, A\&A 445, 1093

Waldron, W. L. \& Cassinelli, J. P. 2001, ApJ, 548, L45

Walborn, N. R., Nichols, J. S., \& Waldron, W. L. 2009, ApJ, 703, 633

Schulz, N. S., Canizares, C. R., Huenemoerder, D. P., \& Lee, J. C. 2000, ApJ, 545, L135

Schulz, N. S., Canizares, C. R., Huenemoerder, D. P., Kastner, J. H., Taylor, S. C., \& Bergstrom, E. J. 2001, ApJ, 549, 441

Schulz, N. S., Canizares, C. R., Huenemoerder, D. P., \& Tibbets, K. 2003, ApJ, 595, 365

Townsley, L. K., Broos, P. S., Feigelson, E. D., Garmire, G., \& Getman, K. 2003, ApJ 593, 874

Townsley, L. K., Broos, P. S., Chu, Y.-H, Gruendl. R. A., Oey, M. S., \& Pittard, J. M. 2011, ApJS, 194, 16

Waldron, W. L. \& Cassinelli, J. P. 2001, ApJ, 548, L45

Walborn, N. R., Nichols, J. S., \& Waldron, W. L. 2009, ApJ, 703, 633

Wood, D. O. S. \& Churchwell, E. 1989, ApJS, 69, 831 